Revista

Actualidades Investigativas

en Educación

\title{
Entre beats y piruetas: Recreación artística y bienestar subjetivo en estudiantes del Colegio Técnico Profesional Ing. Mario Quirós Sasso
}

Between beats and pirouettes: Artistic recreation and subjective wellbeing in students of the Professional Technical College Ing. Mario Quirós Sasso

\section{Volumen 21, Número 1 \\ Enero - Abril \\ pp. $1-30$}

Marilyn Sánchez Fallas

\section{Citar este documento según modelo APA}

Sánchez Fallas, Marilyn. (2021). Entre beats y piruetas: Recreación artística y bienestar subjetivo en estudiantes del Colegio Técnico Profesional Ing. Mario Quirós Sasso. Revista Actualidades Investigativas en Educación, 21(1), 1-30. Doi. 10.15517/aie.v21i1.42454 


\title{
Entre beats y piruetas: Recreación artística y bienestar subjetivo en estudiantes del Colegio Técnico Profesional Ing. Mario Quirós Sasso
}

\author{
Between beats and pirouettes: Artistic recreation and subjective well-being in students of the \\ Professional Technical College Ing. Mario Quirós Sasso
}

\section{Marilyn Sánchez Fallas ${ }^{1}$}

\begin{abstract}
Resumen: El propósito del presente artículo es divulgar los resultados obtenidos del Trabajo Final de Investigación Aplicada (TFIA) de la Maestría Profesional en Recreación de la Universidad de Costa Rica, realizado en el año 2019, cuyo objetivo fue determinar el efecto de participar o no en un programa de recreación artística (danza urbana - Break Dance) en el bienestar subjetivo de estudiantes adolescentes del Colegio Técnico Profesional (CTP) Ing. Mario Quirós Sasso ubicado en el cantón de la Unión, Cartago, Costa Rica. El abordaje del estudio fue cuantitativo de tipo cuasi experimental en el que participaron 34 estudiantes de décimo año, distribuidos en dos grupos intactos, previamente establecidos por la Institución, como grupo control (16 estudiantes) y grupo experimental (18 estudiantes). Ambos grupos completaron la escala de satisfacción con la vida y la escala de balance afectivo (pretest y postest). Con el grupo experimental, se desarrolló un programa de recreación artística de danza urbana de 10 sesiones de 40 minutos por semana. Para analizar los datos, se realizó un Análisis de Varianza (ANOVA) de 2 vías mixto [2 grupos $\times 2$ mediciones] para cada una de las variables. Los resultados demostraron que no hubo diferencias significativas en la variable de satisfacción con la vida, y en la variable de balance afectivo se visualiza una diferencia significativa en ambos grupos en el postest. Se concluyó que la danza es una actividad recreativa artística que prevé a la población adolescente muchos beneficios para su salud física y mental. Sin embargo, este estudio no logró comprobar un beneficio en el bienestar subjetivo de estudiantes adolescentes del CTP Ing. Mario Quirós Sasso luego de participar de un programa en danza urbana.
\end{abstract}

Palabras clave: actividad de tiempo libre, danza, música, calidad de vida.

Abstract: This article communicates the results obtained from a Final Applied Research Project of the Professional Master of Recreation of the University of Costa Rica, carried out during 2019. The objective of the study was to determine the effect of participating or not in an artistic recreation program (urban dance - break dance) in the subjective well-being of adolescent students of the Professional Technical College (CTP) Ing. Mario Quirós Sasso, located in La Unión, Cartago, Costa Rica. The study approach was quasi-experimental quantitative, 34 tenth-year students participated, distributed in two groups previously established by the Institution: a control group (16 students) and an experimental group (18 students). Both groups filled out the life satisfaction scale and the affective balance scale (pretest and posttest); with the experimental group they execute an urban dance artistic recreation program of 10 sessions of 40 minutes per week. To analyze the data, a mixed 2-way Analysis of Variance (ANOVA) [2 groups $x 2$ measurements] was performed for each of the variables. The results showed that there were no differences in the variable of satisfaction with life and in the variable of affective balance, a significant difference was visualized in both groups in the posttest. It is concluded that dance is an artistic recreational activity that establishes many benefits for the adolescent population for their physical and mental health; however, this study does not proof a benefit in the subjective well-being of adolescent students of the CTP Ing. Mario Quirós Sasso that participates in a program of urban dance.

Key words: free time activity, dance, music, quality of life.

\footnotetext{
${ }^{1}$ Labora en la Universidad de Costa Rica como Asesora de proyectos de Acción Social, San José, Costa Rica. Licenciada en Antropología Social y Máster en Recreación de la Universidad de Costa Rica, San José, Costa Rica. ORCID https://orcid.org/00000003-3035-1314?lang=es
}

Dirección electrónica: marilyn.sanchezfallas@ucr.ac.cr, mary8mar@gmail.com

Artículo recibido: 23 de junio, 2020

Enviado a corrección: 15 de setiembre, 2020

Aprobado: 19 de octubre, 2020 


\section{Introducción}

Estudiar los beneficios que prevé la recreación en distintas poblaciones y contextos es de gran relevancia para la promoción de distintas actividades, más aún cuando se trata de poblaciones con alta condición de vulnerabilidad como la adolescente.

La Organización Mundial de la Salud (OMS, 13 de diciembre, 2018) señala que los problemas en la salud mental, específicamente la depresión y el suicidio, afectan a las personas adolescentes de 15 a 19 años, y es la tercera causa de morbilidad y discapacidad de esta población. Este dato sugiere que esta población requiere de intervenciones que contrarresten tales patologías y que contribuyan a mejorar su salud en general. Valga recalcar que, en el contexto costarricense, esta población, usualmente se encuentra cursando la educación secundaria en sistemas formales o abiertos, por lo que el ámbito educativo es un espacio viable para el trabajo con grupos juveniles.

Una de las alternativas a las que se puede recurrir para el abordaje con adolescentes es la recreación. De acuerdo con Salazar (2017), la recreación aporta de manera significativa al bienestar de las personas, promueve la salud y se utiliza para el tratamiento de afecciones o condiciones, así como para la promoción de estilos de vida saludables en personas de cualquier edad. Entre los beneficios obtenidos por la población joven, al participar en actividades artísticas como la música, el drama y la danza, se encuentran mejoras en aspectos como la autoestima, las habilidades sociales, las habilidades artísticas, la confianza y el conocimiento en temas de salubridad, como enfermedades de transmisión sexual, drogas y alcohol (Daykin et al., 2008).

En suma, los beneficios intelectuales o cognitivos que se pueden obtener de la recreación incluyen el aprendizaje de destrezas, valores y comportamientos, y la mejora de la memoria, la atención, la concentración y el pensamiento (Salazar, 2017).

En particular, actividades como la danza permiten que las personas tengan una vida más sana y enérgica, además genera efectos positivos en aspectos psicológicos y contribuye a que las personas jóvenes estén propensas a convertirse en personas adultas activas (Quin, Frazer y Redding, 2007).

Con base en lo expuesto y en relación con los beneficios de la recreación para el bienestar, la condición de vulnerabilidad de la población adolescente y el espacio escolar, como medio para su desarrollo integral, se consideró viable e importante estudiar la percepción del bienestar subjetivo en estudiantes adolescentes antes y después de participar en un programa de recreación artística desde el enfoque de la educación no formal, a partir de la 
valoración de indicadores como la satisfacción con la vida y los afectos positivos y negativos, y su balance.

La satisfacción con la vida se relaciona con la divergencia que percibe la persona entre las aspiraciones personales y sus logros, el rango de evaluación va desde la sensación de realización personal hasta la experiencia de fracaso. Por su parte, el componente de afectividad se expresa en la relación entre emociones positivas y emociones negativas, lo cual se traduce en felicidad (Carballeira, González y Marrero, 2015).

Además, para contribuir en la mejora de la salud integral y la calidad de vida de las personas adolescentes, tanto en términos individuales como sociales, es necesario realizar programas de recreación en el contexto escolar y evaluarlos para medir su impacto. Como efecto colateral, medir el impacto de programas recreativos desarrollados con población estudiantil adolescente permite profundizar en el campo del saber de la recreación y evidenciar las distintas contribuciones, beneficios e impactos que produce inspirado en resultados de índole científico, datos objetivos y procesos de investigación sistemática; asimismo permite hacer del espacio escolar un entorno más atractivo y completo para el desarrollo de la comunidad estudiantil desde una perspectiva holística.

Basado en lo anterior, este artículo pretende divulgar los resultados obtenidos en el Trabajo Final de Investigación Aplicada (TFIA) denominado Recreación artística y bienestar subjetivo en estudiantes adolescentes del Colegio Técnico Profesional Ing. Mario Quirós Sasso, el cual buscó determinar el efecto de participar o no en un programa de recreación artística en el bienestar subjetivo de estudiantes adolescentes de décimo año, y comprobar las siguientes hipótesis:

Ho: No existe una mejora en el bienestar subjetivo en estudiantes adolescentes de décimo año del Colegio Técnico Profesional Ing. Mario Quirós Sasso luego de participar en un programa de recreación artística.

$\mathrm{Hi}$ : Existe una mejora en el bienestar subjetivo en estudiantes adolescentes de décimo año del Colegio Técnico Profesional Ing. Mario Quirós Sasso luego de participar en un programa de recreación artística.

EI TFIA se realizó entre abril y agosto del año 2019, en la sección diurna del CTP Ing. Mario Quirós Sasso ubicado en el cantón de la Unión de la provincia de Cartago, Costa Rica. Este centro educativo ofrece un total de nueve especialidades técnicas y cuenta con una asistencia aproximada de 1650 estudiantes. Para llevar a cabo la investigación se utilizó 
recursos económicos propios, los cuales fueron necesarios para el pago de honorarios de la persona profesional en danza que apoyó en la ejecución del programa de recreación y para el pago de transporte para asistir a la institución educativa.

\section{Referente teórico}

Para comprender los alcances del estudio realizado es indispensable comprender los conceptos asociados a la recreación, como los son el tiempo libre, la recreación artística y sus beneficios. Se explora específicamente las características del Hip Hop y la danza urbana (Break Dance). Asimismo, se abordan aspectos relacionados con la población adolescente, y la importancia del bienestar subjetivo para esta población.

Según Brinnitzer (2003), la definición de tiempo libre depende de las necesidades individuales de la persona, su vivencia y significado de libertad, y sus posibilidades de emplearlo. Por tanto, esta definición parte de una construcción social. Esto quiere decir que está atravesada por una serie de elementos socioculturales definidos por el tiempo y el espacio donde se desarrollan las personas y responde a un determinado momento histórico.

En la actualidad, es usual que el tiempo libre se considere como "una porción de tiempo no dedicada al trabajo, a responsabilidades relacionadas con este, a formas de cuidado personal o a obligaciones familiares y sociales" (Salazar, 2017, p. 8).

Durante el tiempo libre se pueden realizar distintas actividades, pero, por excelencia, es el espacio de tiempo durante el cual se ejerce la recreación. Es importante destacar que para que el tiempo libre sea percibido como tal y lleve a la práctica el sentido de libertad debe acarrear a una experiencia satisfactoria y motivacional en la cual la persona se logre sentir realizada. Caso contrario, sin esta posibilidad, el tiempo libre será un tiempo sin vivencia o de enajenación (Gomes, 2014).

En el contexto sociocultural costarricense, la recreación se comprende como el conjunto de actividades realizadas durante el tiempo libre, que son elegidas de manera voluntaria por las personas y que les resultan agradables, lúdicas, alegres y placenteras (Salazar, 2017). En términos generales, esta práctica se relaciona con aspectos positivos de la vida.

Sobre los beneficios de la recreación, Salazar (2017) menciona que "un beneficio en la recreación es considerado como un logro que demuestra que la participación en la actividad recreativa (1) causó un cambio que se percibió como un mejoramiento o (2) ayudó a mantener una condición deseada" (p. 75). Sin embargo, la misma autora aclara que esta definición queda sujeta a la percepción de las personas y sus condiciones socioculturales, por lo que tiene un 
carácter subjetivo. Estos beneficios pueden clasificarse en términos personales (individuales) y sociales (colectivos), y diferenciarse según las características de las diferentes grupos poblacionales.

De acuerdo con DeGraaf, Jordan y DeGraaf (2010), algunos de los beneficios, a nivel personal, se pueden percibir en términos psicológicos y físicos. Entre los psicológicos se encuentran el crecimiento personal, la autopercepción y la satisfacción personal; y los beneficios físicos se perciben mediante el correcto funcionamiento de los diferentes componentes del cuerpo o a partir de la reducción de enfermedades. Es decir, que las personas gozan de una buena salud física y emocional resultado de integrar en su vida cotidiana las distintas actividades recreativas.

A nivel social, los beneficios de la recreación se reflejan en los resultados positivos de la interacción de las personas en círculos sociales, como la familia, el trabajo, el vecindario, la comunidad y la sociedad como la integración familiar, el clima organizacional, la cohesión social, la disminución de la delincuencia y la protección de los recursos ambientales y culturales (Instituto Colombiano del Deporte [Coldeportes], 2006).

Entre la variedad de actividades recreativas se destaca, en particular, la recreación artística, la cual ofrece experiencias asociadas con la música, la danza, las artes plásticas y manuales, y la actuación o drama. Estas actividades se programan en diferentes formatos como exhibiciones, festivales, conciertos, cursos, entre otros, los cuales se establecen al considerar elementos como el espacio (lugar), la población, los recursos materiales y los objetivos o propósitos planteados (Salazar, 2017).

Vale la pena destacar que la realización de actividades artísticas es muy beneficiosa y positiva, pues fomenta aspectos como la resiliencia, refuerza la autoestima, el autocontrol y la valoración positiva, promueve la creatividad, el humor, la comunicación y las relaciones positivas. Además, permite la expresión y comunicación de emociones por medio de la creación artística (Romero, 2015).

Entre la pluralidad de actividades de recreación artística se destaca la danza, considerada como un arte escénico que involucra la música, el cuerpo y la expresión a partir del movimiento. Es una actividad que reúne elementos físicos y artísticos en su ejecución, por tanto, produce en las personas beneficios en su salud física y psicológica. En el campo de la danza, el movimiento se diferencia del movimiento deportivo al ser un proceso en el cual la persona se sumerge o interioriza en sí misma para experimentar una infinidad de movimientos 
expresivos, creativos y estéticos que reflejan la conexión mente, cuerpo y espacio (Schwender, Spengler, Oedl y Mess, 2018).

Dentro de la danza existe una gran variedad de tipos y expresiones que tienen origen en distintos contextos y épocas. De acuerdo con Bello, Puerta, y Teherán (2016), la danza se define como la alegría de vivir, y es considerada una forma de arte que incorpora el movimiento, el gesto, la comunicación. Además, integra otros elementos que se interrelacionan entre sí, como la expresión corporal, el espacio y el estilo, y suele acompañarse de ritmos musicales y ejecutarse con propósitos de entretenimiento artístico o religioso. Entre los tipos de danza más asociados a los grupos juveniles se encuentra la danza urbana y el Hip Hop, esta es una de las razones que influyó en la escogencia del tipo de danza para desarrollar el programa recreativo.

La danza urbana es un término que se utiliza para referirse a estilos de danza de origen callejero, que surgen de la improvisación y de la comunicación de la persona que baila con el medio donde lo ejecuta. Algunos estilos son el Popping, C-Walk, Locking, entre otros. Sin embargo, está estrictamente vinculada con la cultura del Hip Hop, principalmente con el Break Dance (Bello et al., 2016).

El Hip Hop es un movimiento urbano, artístico y cultural que surge en los barrios populares neoyorquinos de Estados Unidos en la década de 1960 (Merino, 2015). Este movimiento está conformado por cuatro elementos que son muy atractivos y llamativos para ser practicados y observados por la población joven. Estos elementos son el $D j$, el Rap, el Graffiti y el Break Dance (Manotas y Ovalles, 2017).

El Brake Dance es una combinación de acrobacias, influenciado por danzas africanas y capoeira, gimnasia olímpica y artes marciales (Hincapié, 2014). Para Rodríguez e Iglesias (2014), este baile es muy complejo y difícil de realizar debido a la ejecución de ejercicios aeróbicos y gimnásticos, por lo que se requiere disciplina y dedicación. Este baile callejero consiste en la realización de piruetas y movimientos con los pies al ritmo de la música que le propone el $D j$. Esta expresión permite a la población juvenil formar una identidad social y personal de sí mismos, les aleja de malas prácticas (vicios, delincuencia) y les permite crear maneras de ser y actuar en el mundo, por lo que se puede considerar un estilo de vida que promueve valores positivos, como la comprensión, la colaboración y la expresividad; además de características identitarias, tales como la fraternidad, la honestidad y el sentido de superación (Manotas y Ovalles, 2017). 
En específico, el programa de recreación artística de danza urbana ejecutado en el TFIA tiene como base la educación no formal. De acuerdo con Cabalé y Rodríguez (2017), la educación no formal ha existido desde siempre, pero a partir de la década de los 60 es que toma una significancia social debido al incremento de la demanda educativa de la población, los cambios sufridos en la estructura familiar y el aumento del tiempo libre.

Esta modalidad se caracteriza como un conjunto de actividades educativas organizadas y sistémicas que se realizan fuera del sistema formal (oficial), que facilita aprendizajes en diversas temáticas y se dirige hacia diversas poblaciones (Cabalé y Rodríguez, 2017). Sin embargo, como se ha observado en la práctica, en la actualidad, muchas de las actividades educativas no formales se desarrollan dentro de sistemas formales como la escuela, el colegio o la universidad para favorecer y complementar la formación integral de las personas.

En concordancia con lo anterior, se puede afirmar que todas las actividades recreativas se organizan dentro de la educación no formal (Salazar, 2017), pero no todas las actividades de educación no formal son recreativas, pues algunas se dirigen hacia el aprendizaje y actualización de conocimientos para el trabajo.

Ahora bien, con respecto a la población juvenil costarricense, y según la Política Nacional para la Niñez y la Adolescencia (PNNA) (Consejo Nacional de Niñez y Adolescencia, 2009), el Código de la Niñez y Adolescencia (CNA) establece que la adolescencia es una etapa que se encuentra entre la niñez y la adultez, dentro del rango etario de los 12 a los 18 años, que se caracteriza por cambios físicos y anímicos. Entre los derechos más relevantes que tiene esta población se encuentran: vivir con su familia, mantenerse dentro de los sistemas educativos formales e informales y ejercer su autonomía para la construcción de su identidad.

Los cambios y los riesgos que afectan la integridad de la población adolescente, en específico, a la salud mental, y como lo señaló la OMS, se relacionan esencialmente con la conducta y las emociones. Esta etapa, por lo tanto, también tiene implicaciones sobre el bienestar subjetivo, el cual se asocia con la satisfacción por la vida y la resiliencia, emociones necesarias para el afrontamiento de los riesgos asociados (Rodríguez-Fernández et al., 2016).

En relación con el bienestar, este es un constructo complejo que se encuentra vinculado a nociones más amplias, como la psicología positiva, y puede entenderse en dos ámbitos: subjetivo y psicológico. Esta rama de la psicología se enfoca en estudiar temáticas y procesos sobre funcionalidad y ajuste psicológico positivo que impacta sobre la calidad de vida. Además, considera otros aspectos como el apoyo social, el estándar de vida y la salud física, y toma como apoyo los contenidos de otras disciplinas como la sociología (San Martín, 2011). 
Para efectos de este artículo se enfatizó en el bienestar subjetivo, San Martín (2011) señala que se caracteriza como un "estado de felicidad, relajación y ausencia relativa de problemas, el bienestar eudaimónico se asocia a ser desafiado, esforzarse y buscar el crecimiento y desarrollo personal" (p. 20). Además, se refiere a la valoración que realizan las personas sobre algunos aspectos de su vida, tales como su cuerpo, circunstancias en las que viven y los acontecimientos sucedidos. Se relaciona con la percepción de la experiencia positiva y negativa, y las respuestas emocionales generadas ante diversas circunstancias, las cuales pueden observarse a nivel general o en dominios específicos, como el estudio o la recreación. En síntesis, se refiere a la evaluación cognitiva que realiza el ser humano sobre la satisfacción con la vida (Sandoval, 2014).

De acuerdo con Laca (2015), el concepto de bienestar subjetivo fue acuñado a partir de 1980 por el psicólogo estadounidense Edward F. Diener para comprender cómo las personas experimentan de manera positiva la vida con base en aspectos cognitivos y emocionales o afectivos a partir de su propio conjunto de criterios y prioridades personales; es decir, desde la subjetividad como individuo.

El bienestar subjetivo se analiza desde tres componentes: (1) la satisfacción con la vida, el cual es cognitivo; (2) el afecto o emoción positiva y negativa, estos dos componentes de naturaleza emocional se relacionan entre sí, pero son independientes uno de otro; (3) se analiza desde el componente global o específico, y profundiza en algunos dominios de la vida o en algunos afectos (Sandoval, 2014).

La variable de la temporalidad es de gran importancia en el bienestar subjetivo, principalmente en la relación con los estados afectivos positivos y negativos, en los cuales la frecuencia tiene mayor impacto que la intensidad. El componente de satisfacción con la vida se considera más estable porque su valoración no es susceptible a cambios a corto plazo (Sandoval, 2014).

Según Lara (2017), la felicidad depende más de predisponentes personales que de eventos externos, aunque, según sea la carga afectiva de estos, pueden incidir en los estados de ánimo que después volverán a su estado inicial o característico. Este autor propone que el bienestar subjetivo no depende de aspectos materiales ni económicos, sino más bien de aspectos sociales y relacionales.

En este sentido, la experiencia positiva que producen las actividades recreativas en las personas puede influenciar en la percepción que tienen sobre su propia felicidad e, incluso, moldear estilos de vida que contribuyen a fortalecer rasgos personales (Lara, 2017). Esto 
quiere decir que las actividades recreativas pueden hacer que las personas se sientan más felices y, en consecuencia, reduzcan emociones o comportamientos negativos, como el enojo o la tristeza.

En síntesis, los beneficios de la recreación, específicamente los generados por la danza urbana en ámbitos educativos y con población adolescente, pueden reflejarse en aspectos como la percepción de satisfacción con la vida y la felicidad, aspectos de mucha relevancia para el desarrollo integral y la calidad de vida de todo ser humano.

\section{Metodología}

\subsection{Enfoque}

La investigación se desarrolló bajo el enfoque cuantitativo y de tipo cuasi experimental debido a que fueron seleccionados grupos intactos de similar condición. La pregunta que fundamentó el abordaje fue ¿cuál es la influencia que tiene un programa de recreación artística en el bienestar subjetivo de estudiantes adolescentes de décimo año del Colegio Técnico Profesional Ing. Mario Quirós Sasso? Se plantearon dos hipótesis sobre si existe o no una mejora en el bienestar subjetivo en estudiantes adolescentes luego de participar o no en un programa de recreación artística. Los grupos fueron determinados por la Institución como el grupo control y experimental según la conveniencia del horario académico, con el fin de que no se viera afectado el programa de estudios.

Se realizó un trabajo de investigación aplicada a la población participante en la cual se realizó una medición inicial (pretest) para medir el bienestar subjetivo (variable dependiente) a partir de la escala de satisfacción con la vida y la escala de balance afectivo. Seguidamente, se ejecutó un programa de recreación artística de danza urbana y, al finalizar, se realizó una segunda medición de la variable indicada (postest).

\subsection{Unidades de análisis}

El trabajo de campo se realizó de mayo a agosto del año 2019 con dos grupos de estudiantes adolescentes de décimo año del Colegio Técnico Profesional Ing. Mario Quirós Sasso. El grupo control y el experimental (grupos intactos) asignados estaban conformados por 19 y 20 estudiantes, respectivamente.

Se solicitó a la Institución la posibilidad de asignar el tratamiento de manera aleatoria a los grupos. Sin embargo, por directrices de la Institución, desde el inicio, se condicionó a que el programa se realizaría durante la lección de Orientación y Guía. Por tanto, la Orientadora 
encargada determinó el grupo experimental. El grupo control fue seleccionado por la misma profesora contemplando la misma cantidad de estudiantes y similares condiciones que el grupo experimental (rangos de edad).

Para la selección del estudiantado se tomó en cuenta los siguientes criterios de inclusión y exclusión:

\subsubsection{Criterios de inclusión}

- Dentro de los grupos de décimo año solo se tomaría en cuenta el estudiantado con edades entre 15 y 19 años.

- Que, al menos, el $80 \%$ del estudiantado de cada grupo se encontrara anuente a participar en la investigación.

- Que el estudiantado participara en, al menos, el $80 \%$ de las sesiones del programa de recreación artística.

\subsubsection{Criterios de exclusión}

- Estudiantes que se encontraban practicando danza urbana en el momento de la investigación.

- Estudiantes que se encontraban tomando algún medicamento que pudiera alterar su estado anímico.

- Estudiantes menores de 15 años y de 20 años o más.

En total, se tomaron en cuenta para el estudio 18 estudiantes del grupo experimental (según criterios de inclusión) y se excluyeron dos debido a que un estudiante solo asistió a tres sesiones ( $30 \%$ de asistencia) y otra estudiante no entregó el consentimiento firmado. Asimismo, del grupo control se excluyeron tres estudiantes (dos estudiantes no completaron el cuasi experimento y una no entregó el consentimiento), por lo que la muestra total fue de 16 estudiantes.

Ambos grupos completaron el consentimiento informado, el cual fue redactado siguiendo los lineamientos del Comité Ético Científico de la Universidad de Costa Rica y aprobado en la sesión número 138 celebrada el 03 de abril del 2019. 


\subsection{Técnicas de recolección}

Las escalas utilizadas para la medición de la variable fueron dos. Por un lado se aplicó la Escala de Satisfacción con la Vida (Satisfaction with Life Scale- SWLS) adaptada al español (castellano) por Atienza, Pons, Balaguer y García-Merita (2000), quienes analizaron las propiedades psicométricas de la versión en castellano de la escala en una muestra de adolescentes valencianos según la estructura factorial, la consistencia interna y la validez del constructo, fue satisfactoria. De acuerdo con Gutiérrez, Tomás y Calatayud (2017)

Esta escala ha aportado evidencias de validez y fiabilidad en numerosos países y con muestras de diferentes edades y condiciones sociales, por ejemplo la de Atienza et al. (2000) con adolescentes españoles y portugueses, y la de Núñez, Martín y Domínguez (2010) con muestras de adultos españoles practicantes de actividad física. (p. 160)

De acuerdo con Atienza et al (2000), el análisis de fiabilidad de la escala en versión al español posee una buena consistencia interna y una validez convergente adecuada. El coeficiente alfa de Cronbach para la escala completa, obtenido en algunos estudios, oscila entre 0,89 y 0,79 .

Este instrumento está compuesto por cinco ítems que proporcionan un índice general de satisfacción con la vida a partir de una escala tipo Likert que va de 1 a 7 (1 = "Totalmente en desacuerdo" a 7 = "Totalmente de acuerdo") (Godoy-Izquierdo, Martínez y Godoy, 2008; Gutiérrez et al., 2017). Según Barrantes-Brais y Ureña-Bonilla (2015), la puntuación puede analizarse de manera continua o puede clasificarse en intervalos de: 30-35 (altamente satisfecho); de 25-29 (satisfecho); de 20-24 (ligeramente satisfecho); de 15-19 (ligeramente insatisfecho), de 10-14 (insatisfecho), y de 5-9 (muy insatisfecho). Este instrumento es completado por la persona en un lapso de tres minutos. Ver escala en Anexo A.

Por otro lado se aplicó la Escala de Balance Afectivo (EBA) adaptada al español (castellano) por Godoy et al (2008), quienes confirman que "la escala muestra una adecuada validez convergente con medidas de competencia personal inespecífica o global, de salud física y mental y de satisfacción vital" (p. 157).

Está compuesta por 18 ítems (nueve para evaluar afectos positivos y nueve para afectos negativos). Los coeficientes de fiabilidad alfa de Cronbach obtenidos en el estudio de Rodríguez et al (2016) fueron de 0,80 en afecto negativo y 0,78 en afecto positivo. 
Al completar la escala, las personas deben indicar si han experimentado en la última semana los estados que se enlistan en el instrumento. La pregunta se contesta con una escala tipo Likert de 3 alternativas: 1 (poco o nunca), 2 (a veces) y 3 (mucho o generalmente).

La escala mide el afecto positivo y el negativo en puntuaciones de 9 a 27 . Además, se obtiene un índice de balance afectivo total que se calcula con base en la diferencia resultante entre el afecto positivo y el afecto negativo (-18 a 18 puntos). Los valores negativos a partir de cero indican un balance afectivo negativo mientras que los valores positivos por encima de cero, un balance afectivo positivo. Este instrumento es completado por la persona en un lapso de cinco minutos. Ver escala en Anexo B.

Los dos instrumentos utilizados se encuentran validados para adolescentes y en idioma español, sin embargo, se efectuó un plan piloto para verificar que el estudiantado adolescente comprendiera los enunciados de ambos instrumentos y así obtener los coeficientes de confiabilidad para la muestra de estudio.

El plan piloto se realizó con una muestra de 46 estudiantes con edades de 15 a 19 años que cursaban otros grupos de décimo año del CTP. A cada estudiante se le solicitó completar el instrumento e indicar si tenía alguna observación o pregunta sobre los enunciados. El propósito de esta acción fue validar que el instrumento era comprensible para la población participante. Solo una estudiante sugirió hacer inclusivo cada uno de los ítems del instrumento. Es preciso aclarar que las personas participantes del plan piloto no fueron las mismas personas de la muestra de investigación.

Junto con las escalas que se aplicaron para valorar las variables independientes se le solicitó al estudiantado del grupo control y del grupo experimental completar un instrumento para recopilar información sociodemográfica y actividades recreativas artísticas que realizaban. La información se recolectó tanto en el pretest como en el postest.

Utilizar este instrumento permitió identificar las respuestas de las personas participantes en el pretest y en el postest para el análisis de los datos. A las personas participantes del grupo experimental que señalaron que realizaban alguna actividad artística relacionada con el baile, se les preguntó de manera verbal, en el postest, si el programa de danza urbana ofrecido era igual o similar a las actividades de baile o danza que realizaban en otro momento. Al respecto, todas las respuestas fueron negativas; es decir, ninguna de las actividades recreativas artísticas que las personas participantes realizaban eran como las desarrolladas en el programa de danza urbana. 
Se ejecutó un programa educativo de recreación artística con el grupo experimental. Inicialmente, se definió realizar un programa de catorce semanas, una sesión por semana de 40 minutos durante las lecciones de Guía y de Orientación, según había establecido el centro educativo. Sin embargo, por disposiciones posteriores de la Institución, solamente se lograron realizar 10 sesiones. El programa consistió en explicar al estudiantado el origen de la danza urbana y sus características, así como en demostrar los pasos o movimientos básicos y su significado corporal. Este programa fue desarrollado por un bailarín especialista en danza urbana, con más de 20 años de experiencia y que había trabajado en programas similares con población juvenil.

La población estudiantil adolescente que recibió el programa prestó atención a las explicaciones e indicaciones brindadas por parte de la investigadora (quien coordinó, programó y evaluó la actividad) y de la persona especialista en danza (quien ejecutó la actividad). Asimismo, la población estudiantil observó demostraciones y participó de forma pasiva (sin bailar), pero siempre interactuando con el grupo, o activa (bailando) en la actividad planteada.

A los dos grupos (control y experimental) se les solicitó que completaran las escalas antes de iniciar el programa de recreación artística, el cual solo se realizó con el grupo experimental. Al finalizar el programa se solicitó nuevamente a los grupos completar las escalas.

El grupo experimental completó el pretest en el gimnasio del centro educativo donde se realizó la primera sesión, seguidamente se ejecutó el desarrollo de la primera sesión del programa. El postest fue completado al final de la última sesión del programa en el aula multiusos. Por su parte, el grupo control completó el pretest y postest durante la clase de Orientación una semana después de la aplicación al grupo experimental.

Para completar las escalas, se solicitó al estudiantado marcar con lápiz o bolígrafo, de manera individual, la opción que consideró que más se ajustaba a su respuesta. El procedimiento de recolección de información y puesta en práctica del programa de recreación se realizó durante mayo y agosto del año 2019.

\subsection{Procesamiento de análisis}

Para obtener los puntajes de cada una de las escalas, se sumaron los ítems obteniendo un total. Además, en la EBA se calculó la diferencia entre el afecto positivo y el afecto negativo para obtener el balance afectivo total. En cuanto a la estadística descriptiva, se obtuvieron los 
promedios y desviaciones estándar de las personas participantes en relación con sus características principales (edad, sexo) y los puntajes de escala 1 y escala 2, para lo cual se utilizó el programa Excel 2016.

Por otra parte, la estadística inferencial consistió en un Análisis de Varianza (ANOVA) de 2 vías mixto ( 2 grupos $\times 2$ mediciones) con medidas repetidas en el último factor. En este caso se empleó el programa estadístico SPSS (versión 20,0). Se realizó un ANOVA para cada una de las variables dependientes, y así determinar el efecto del programa de recreación artística de danza urbana según la escala de satisfacción con la vida y la de balance afectivo. El análisis de normalidad y homocedasticidad indicó que las variables presentan una distribución normal y su varianza es homogénea $(p>, 05)$.

\section{Resultados}

La estadística descriptiva muestra el total de estudiantes adolescentes que conformaron el estudio según sexo y edad. En la Tabla 1 se observa la cantidad de estudiantes que se tomaron en cuenta en el estudio según los criterios de inclusión definidos.

Tabla 1

Total de estudiantes participantes del CTP Ing. Mario Quirós Sasso por grupo y según sexo, 2019

\begin{tabular}{lrrr}
\hline \multicolumn{1}{c}{ Participantes } & GC & GE & Total \\
\hline Cantidad de estudiantes de la muestra & 16 & 18 & 34 \\
Cantidad de hombres & 2 & 13 & 15 \\
Cantidad de mujeres & 14 & 5 & 19 \\
\hline
\end{tabular}

Fuente: Elaboración propia, 2019.

Nota: $\mathrm{GC}=$ Grupo Control, GE = Grupo Experimental.

En la Tabla 2 se muestra el promedio y la desviación estándar de la edad de cada grupo. Se puede observar que la población participante, en general, se encontraba entre los 15 y 16 años.

Tabla 2

Estadística descriptiva del estudiantado participante del CTP Ing. Mario Quirós Sasso según edad, 2019

\begin{tabular}{lll}
\hline Grupo & $\mathrm{M}$ & $\mathrm{DE}$ \\
\hline $\mathrm{GC}$ & 15,69 & 0,70 \\
$\mathrm{GE}$ & 16,17 & 0,92 \\
\hline
\end{tabular}

Fuente: Elaboración propia, 2019.

Nota: $\mathrm{GC}=$ Grupo Control, GE = Grupo Experimental, $\mathrm{M}=$ Media, $\mathrm{DE}=$ Desviación Estándar. 
En relación con los resultados de las dos variables dependientes del estudio, en la Tabla 3 se observa que en la variable de satisfacción con la vida, ambos grupos se encuentran dentro de la clasificación de "satisfecho(a)", la cual contempla un rango de puntuación entre los 25 y 29 puntos tanto en el pretest como en el postest. En cuanto al balance afectivo se muestra que, en promedio, el balance es positivo, pues no hay valores negativos ya que los resultados obtenidos están por encima de cero.

Tabla 3

Resultados de la estadística descriptiva del estudiantado participante del CTP Ing. Mario Quirós Sasso según la variable de satisfacción con la vida y de balance afectivo, 2019

\begin{tabular}{|c|c|c|c|c|c|c|c|c|}
\hline \multirow{4}{*}{ Grupo } & \multicolumn{8}{|c|}{ Variables } \\
\hline & \multicolumn{4}{|c|}{ SV } & \multicolumn{4}{|c|}{ BA } \\
\hline & \multicolumn{2}{|c|}{ Pre } & \multicolumn{2}{|c|}{ Post } & \multicolumn{2}{|c|}{ Pre } & \multicolumn{2}{|c|}{ Post } \\
\hline & $M$ & $\mathrm{DE}$ & $M$ & $\mathrm{DE}$ & $\mathrm{M}$ & $\mathrm{DE}$ & $M$ & $\mathrm{DE}$ \\
\hline GC & 25,81 & 7,70 & 27,63 & 6,21 & 1,50 & 7,52 & 3,38 & 6,02 \\
\hline GE & 27,28 & 4,96 & 27,39 & 4,88 & 4,28 & 4,91 & 6,39 & 5,18 \\
\hline
\end{tabular}

Fuente: Elaboración propia, 2019.

Nota: GC = Grupo Control, GE = Grupo Experimental, SV = Satisfacción con la Vida, BA = Balance Afectivo, $\mathrm{M}=$ Media, $\mathrm{DE}=$ Desviación Estándar.

Al desagregar la variable de balance afectivo se obtienen nueve afectos positivos y nueve afectos negativos. En la Tabla 4 se observa la puntuación de los niveles encontrados según el tipo de afecto (positivo o negativo) en el grupo control, y en el grupo experimental según la medición (pre y post). Se observó que en ambos grupos la puntuación de los afectos positivos es mayor a 20 , lo cual es relativamente alta si se toma como referencia que el mayor puntaje posible es de 27.

Tabla 4

Niveles del balance afectivo del estudiantado participante del CTP Ing. Mario Quirós Sasso según el tipo de afecto (positivo o negativo) y la medición (pre y post), 2019

\begin{tabular}{lcccccccc}
\hline & \multicolumn{3}{c}{ Afectos positivos } & \multicolumn{3}{c}{ Afectos negativos } \\
\cline { 2 - 10 } Grupo & \multicolumn{3}{c}{ Pretest } & \multicolumn{3}{c}{ Postest } & \multicolumn{3}{c}{ Pretest } & \multicolumn{2}{c}{ Postest } \\
\cline { 2 - 10 } & $M$ & DE & $M$ & DE & M & DE & M & DE \\
\hline GC & 20,44 & 3,52 & 21 & 2,50 & 18,94 & 4,89 & 17,63 & 4,59 \\
GE & 21,89 & 2,47 & 22 & 2,63 & 17,61 & 3,31 & 15,61 & 3,70 \\
\hline
\end{tabular}

Fuente: Elaboración propia, 2019.

En cuanto a la estadística inferencial, en la variable de Satisfacción con la Vida, se encontró que no existen diferencias significativas en el efecto simple de grupo, ni en el efecto simple de medición y ni tampoco se encontró interacción significativa, por tanto no fue necesario realizar un análisis post-hoc (ver Tabla 5). 
Tabla 5

Resultados de la variable Satisfacción con la Vida del estudiantado participante del CTP Ing. Mario Quirós Sasso, 2019

\begin{tabular}{lrl} 
& $F$ & $P$ \\
\hline Grupo & 0,105 & 0,748 \\
Medición & 1,48 & 0,231 \\
Grupo x medición & 1,16 & 0,289 \\
\hline
\end{tabular}

Fuente: Elaboración propia, 2019.

En la Figura 1 se muestra la comparación entre el promedio total de las mediciones de los dos grupos. Se logra observar que hubo un aumento en la satisfacción con la vida, pero no fue significativo.

Figura 1

Promedio de la variable de satisfacción con la vida según grupos y mediciones del estudiantado participante del CTP Ing. Mario Quirós Sasso, 2019

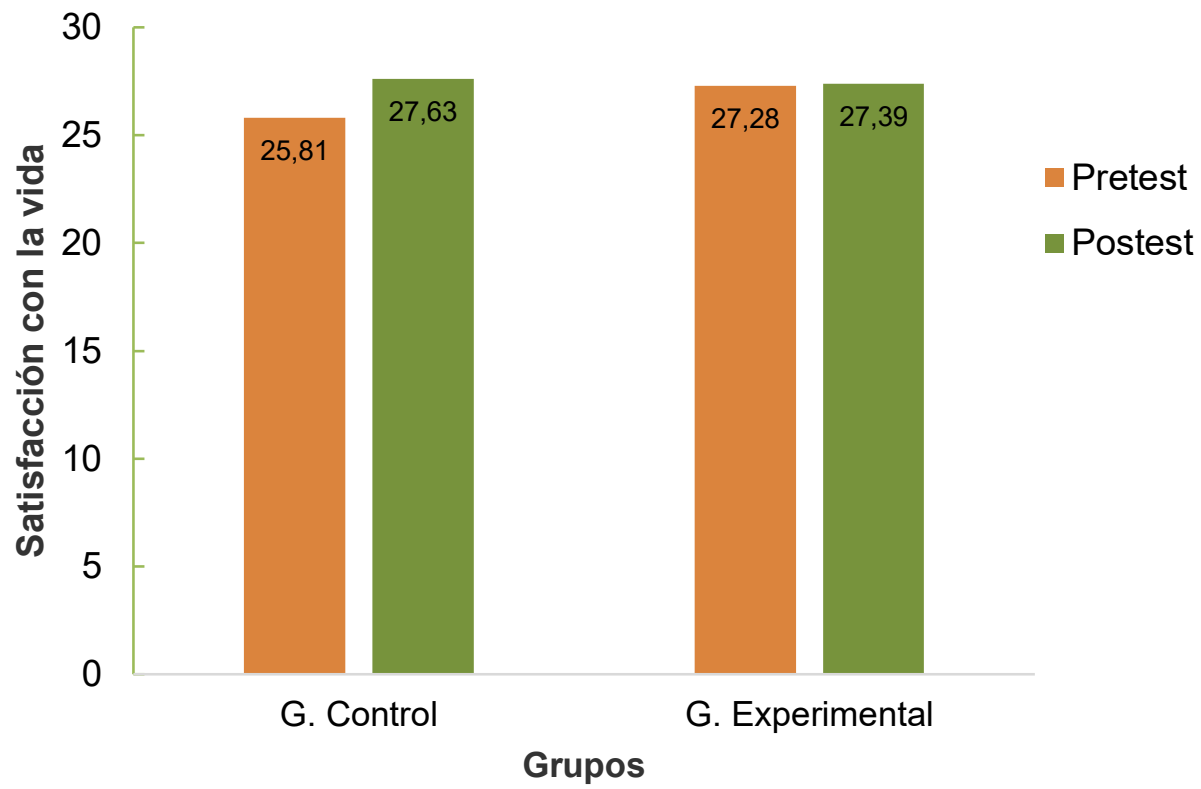

Fuente: Elaboración propia, 2019.

Por su parte, la variable de balance afectivo se compone de la diferencia resultante entre la puntuación del afecto positivo y la puntuación del afecto negativo; sin embargo, es importante observar los niveles de afectos positivos y negativos de manera independiente.

En el análisis de esta variable se encontró que existe una diferencia significativa entre las mediciones $(p=, 004)$, mas no existe diferencia en el efecto simple de grupo ni en la interacción de grupo x mediciones (ver Tabla 6). Es decir, que en el postest las puntuaciones de las escalas aplicadas de ambos grupos mejoraron. 
Tabla 6

Resultados de la variable Balance Afectivo del estudiantado participante del CTP Ing. Mario Quirós Sasso, 2019

\begin{tabular}{lcc}
\hline \multirow{2}{*}{ Grupo } & $F$ & $P$ \\
\cline { 2 - 3 } & 2,23 & 0,114 \\
\hline Medición & 9,49 & $0,004^{*}$ \\
Grupo x medición & 0,03 & 0,856 \\
\hline
\end{tabular}

Fuente: Elaboración propia, 2019.

En la Figura 2 se observa la interacción de los grupos y las mediciones, lo que demuestra que el balance afectivo de ambos grupos aumentó en la segunda medición. En el caso del grupo Control el resultado obtenido pasó de 1,50 a 3,38 y para el grupo Experimental aumentó de 4,28 a 6,39 .

Figura 2

Interacción de los grupos y mediciones de la variable de balance afectivo del estudiantado participante del CTP Ing. Mario Quirós Sasso, 2019.

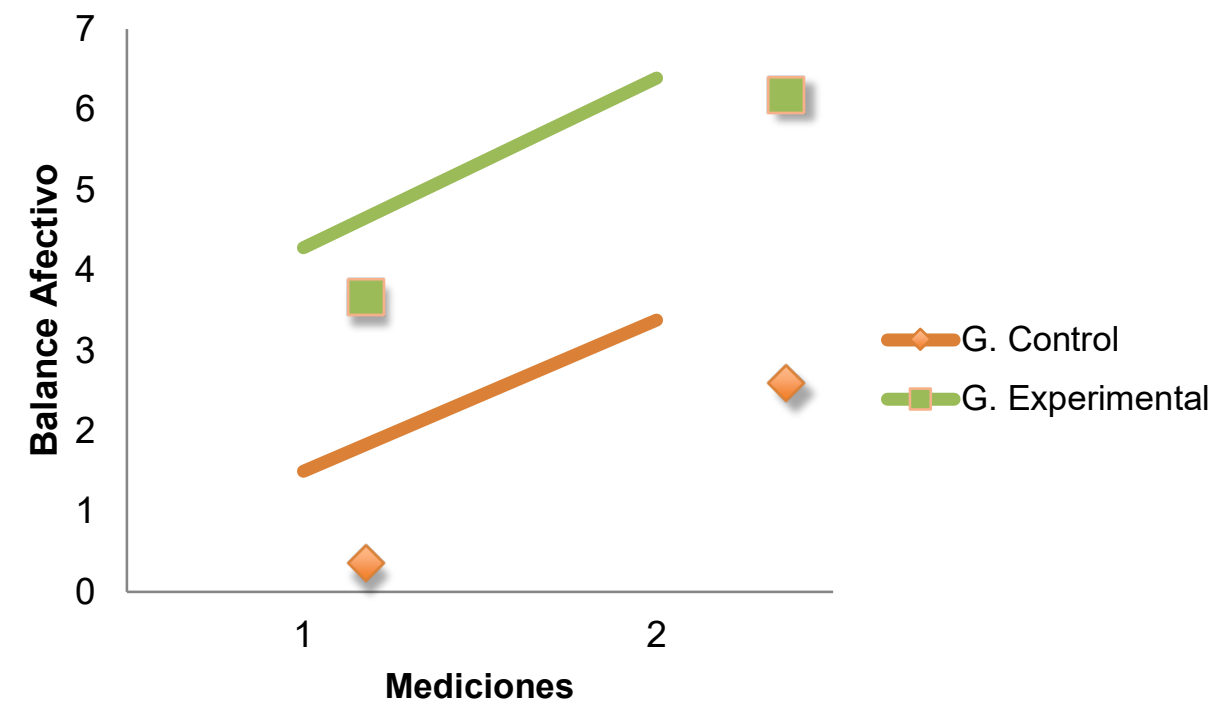

Fuente: Elaboración propia, 2019.

Los resultados de este estudio demostraron que no se puede atribuir un efecto en el bienestar subjetivo de la población de estudiantes adolescentes luego de participar en un programa de recreación artística de danza urbana, pues la variable de satisfacción con la vida no muestra diferencias significativas y la variable de balance afectivo es significativa para ambos grupos.

Si bien este estudio no evidenció un efecto diferente en el bienestar subjetivo entre el grupo control y el grupo experimental, algunas investigaciones han demostrado cómo la danza 
contribuye a mejorar variables asociadas a la salud mental en grupos poblacionales, tales como la niñez, adolescencia, adultez y adultez mayor.

En este sentido, Padilla y Coterón (2013) destacan que la danza ha contribuido a mejorar aspectos como la salud, la capacidad funcional, las relaciones sociales, el bienestar material, personal y social, y otros relacionados con emociones negativas como la depresión, la ansiedad, la hostilidad, la obsesión, las ideas paranoicas y psicóticas en poblaciones de mujeres adolescentes con problemas psicológicos. Además, plantean que existe una carencia de estudios sobre la danza y sus beneficios en poblaciones sin problemas asociados a la salud mental.

Schwender et al (2018) señalan que, a pesar de los resultados positivos de las intervenciones de danza en diferentes grupos poblacionales en distintos aspectos, la mayoría de estudios son muy heterogéneos en cuanto a la muestra, la metodología aplicada, las intervenciones de danza y las variables en estudio, por lo que es difícil determinar de manera más profunda los posibles beneficios de la danza.

Ramos-Díaz, Rodríguez-Fernández y Antonio-Aguirre (2017) analizan las mismas dos variables que se utilizaron en esta investigación para determinar el bienestar subjetivo. Además, analizaron la correlación con la variable de autoconcepto (esta variable hace referencia al concepto que tienen las personas sobre sí mismas, además esta variable se corresponde con la variable de la felicidad). Entre los resultados obtenidos observaron que estudiantes con alto autoconcepto presentan mayores puntuaciones en satisfacción con la vida y afecto positivo, y puntuaciones más bajas en afecto negativo en comparación con estudiantes que manifiestan un bajo autoconcepto.

Según los resultados obtenidos en este estudio, tanto en el pretest como en el postest, el grupo control y el grupo experimental se encontraban dentro del rango de "satisfecho" en una clasificación que comprende seis rangos en su totalidad (variable de satisfacción con la vida). De acuerdo con Gutiérrez et al (2017), este es un indicador relevante de la salud mental y el bienestar subjetivo en la adolescencia. Asimismo, en la variable de balance afectivo, la puntuación de los afectos positivos obtenida fue alta. Por tanto, el nivel del estado de bienestar subjetivo de la población participante de toda la muestra limita obtener cambios significativos en vista de que el nivel inicial de la población era alto.

Romero (2010) apunta a que se puede promover la actividad física y la recreativa para tratar problemáticas contemporáneas en poblaciones jóvenes, como la obesidad. Además, la danza, específicamente el Hip Hop, es una estrategia para afrontar las barreras de los 
contextos residenciales de bajos ingresos, de tal manera que la juventud pueda tener mayor acceso y disponibilidad de recursos para realizar actividad física y recreativa.

Del trabajo de Romero (2010) se rescata la similitud sobre la aplicación de 10 sesiones de un programa de Hip Hop. Sin embargo, la metodología empleada en el programa es diferente a la utilizada en esta investigación, así como las edades de la población participante y las variables analizadas. Otro elemento similar fue que se realizó un pretest antes de iniciar el programa y un postest luego de concluir la intervención.

Para Zapata (2016), una explicación que justifica por qué la danza puede influir en la percepción del bienestar psicológico y social es que es un tipo de actividad física y como tal, conlleva una serie de beneficios en cuanto a la salud y el bienestar de las personas, que han sido comprobados con fundamento científico. Por otra parte, señala que el contenido social y cultural de algunos tipos de danza promueve vínculos sociales positivos y la participación en espacios socioculturales. También, pueden otorgar estructuras psicológicas de identificación y sentido de pertenencia con el entorno, lo cual, en suma, contribuye al bienestar de las personas que practican danzas, como la folklórica.

Asimismo, los beneficios de la danza son observables en otros aspectos de la población adolescente, como la calidad de vida y la disminución en gastos médicos. Philipsson, Duberg, Möller y Hagberg (2013) señalan que, a partir de una intervención en danza, se obtuvo una mejora en la calidad de vida de mujeres adolescentes que les permitió disminuir la atención médica. No obstante, el estudio en mención no muestra similitudes con esta investigación, pues el programa de danza que ejecutó es de mayor duración (ocho meses) y la población de adolescentes (de 13 a 18 años) solo incluye femeninas con problemáticas psicosociales.

Connolly, Quin y Redding (2011) han demostrado que al participar en un programa (Dance 4 for life project) de cinco a doce horas (seis semanas) se puede obtener un impacto positivo en adolescentes femeninas en términos del bienestar fisiológico y psicológico. Estos autores resaltan la importancia de la motivación intrínseca y el disfrute de la actividad para que los resultados sean positivos. En comparación con esta investigación, se destaca que el contexto donde se desarrolla el programa de danza es de índole escolar y, por tanto, algunas de las jóvenes participaron por obligación, mientras que otras mostraron una gran motivación intrínseca.

De los estudios antes mencionados se concluye que no hay referencia de estudios que analicen el bienestar subjetivo en dos de sus dimensiones de análisis (satisfacción con la vida y balance afectivo) en relación con la aplicación de un programa de recreación artística de 
danza urbana en población adolescente. Sin embargo, la danza como actividad de recreación artística sí proporciona a las personas efectos positivos (Connolly et al., 2011; Philipsson et al., 2013; Romero, 2010; Schwender et al., 2018; Zapata, 2016), aunque en el caso de esta investigación, no se logró demostrar ningún efecto positivo.

Finalmente, se reitera que la totalidad de la población participante en este estudio mostró, desde el inicio y hasta el final del tratamiento, un estado de bienestar subjetivo satisfactorio o deseable, lo cual puede indicar que la población goza de un buen estado emocional. Además, a diferencia de otros estudios, en este abordaje la población participante no se considera con problemáticas riesgosas o prioritarias de atención que requieran modificarse a partir de una intervención o tratamiento.

\section{Conclusiones}

Los resultados obtenidos señalan que en esta investigación se cumplió la hipótesis nula, pues no se puede asegurar que existió una mejora en el bienestar subjetivo de estudiantes adolescentes después de participar en un programa de recreación artística de danza urbana, por cuanto no hubo una diferencia estadísticamente significativa en el nivel de bienestar subjetivo.

En cuanto al cumplimiento de los objetivos planteados, no se logró determinar que hubo un efecto en el bienestar subjetivo en el grupo control de estudiantes adolescentes de décimo año del CTP Ing. Mario Quirós Sasso en ninguna de las dos variables que se pudiera atribuir al programa de recreación artística de danza urbana; debido a que en ambos grupos, tanto el experimental como el control, se observó un aumento, principalmente, en la variable de balance afectivo.

Se rescata que los valores obtenidos en la primera medición (pretest) de ambos grupos muestran altos niveles de puntuación, tanto para la variable de satisfacción con la vida como para la variable de balance afectivo. Por un lado, esta condición inicial limitó que existiera un aumento en la segunda medición luego de participar en un programa de danza urbana, pues los valores encontrados inicialmente se consideran satisfactorios o deseables.

Por otro lado, de acuerdo con Salazar (2017), se puede considerar que sí hubo un beneficio por cuanto se mantuvo la condición deseada en el grupo experimental luego de participar en el programa recreativo. Aunado a lo anterior, ambos grupos se mostraron activos en la realización de actividades recreativas artísticas variadas, las cuales desarrollan a lo largo de su cotidianidad fuera o dentro del centro educativo. 
Que la población realizara actividades recreativas pudo haber influido en su estado de bienestar subjetivo, pues ya obtienen los beneficios de la recreación. La significancia encontrada en la variable de balance afectivo en ambos grupos coincide con lo que Sandoval (2014) señala sobre la variable de la temporalidad en el bienestar subjetivo, ya que, en la relación de los estados afectivos positivos y negativos la frecuencia tiene mayor impacto que la intensidad pero en la satisfacción con la vida no es susceptible a cambios a corto plazo; en esta última variable no se registró una diferencia significativa.

Además, según Lara (2017), si los eventos externos provocan una carga afectiva en la persona, los estados de ánimo pueden verse alterados, pero después volverán a retornar a su estado característico. Quiere decir que no existe un efecto prolongado en los afectos si se considera que son parte de los estados de ánimo y que, además, pueden variar de un momento a otro según lo que experimente la persona.

En el caso particular de las actividades recreativas, estas siempre provocarán una experiencia positiva, lo que puede alterar el estado de ánimo como la felicidad; sin embargo, en este estudio no se logró demostrar estadísticamente un efecto significativo. De acuerdo con DeGraaf et al (2010), el disfrute y la diversión que obtienen las personas resultado de su participación en las actividades recreativas se considera como el aporte de la recreación en el bienestar.

En este sentido, se rescata que, como una etapa del programa de recreación, se programó la aplicación de una evaluación que valoró la opinión de la población participante. Los resultados obtenidos de la evaluación mostraron que, a partir del desarrollo del programa de danza urbana, el grupo de estudiantes, en su mayoría, experimentó diversión durante las sesiones.

En relación con las contribuciones que la investigación aportó al ámbito de lo educativo, estas se puede expresar en dos dimensiones: desde la educación formal y la no formal. Se rescata que la población estudiantil que participó en el programa de recreación artística tuvo la oportunidad de aprender, dentro del centro educativo, movimientos y contenidos de la danza urbana, y ponerlos en práctica a partir de la mediación de un profesional reconocido y de manera gratuita.

Esta experiencia permitió a las personas participantes obtener los beneficios de la recreación antes mencionados, disfrutar con sus pares de la interacción de una actividad de educación no formal, creativa, liberadora y expresiva dentro del centro educativo, lo que en alguna medida puede mejorar la convivencia escolar. 
Por otra parte, implementar programas recreativos dentro de instituciones educativas previene o facilita la atención de problemáticas como el bullying, lo anterior tomando en cuenta que la recreación se desarrolla desde enfoques multidisciplinarios dirigidos a modificar aspectos del ambiente o de la cultura, fomentando valores como la cooperación, la confianza, la resiliencia, el respeto y la tolerancia (Salazar, 2017).

Entre las fortalezas de esta investigación se destaca que utilizar una metodología cuasi experimental en la cual se determina un grupo control y otro experimental, se aplican varias mediciones y se ejecuta un tratamiento recreativo, permite observar de manera estadística y objetiva las contribuciones que puede generar la recreación. Aunque para el caso del TFIA no se logró demostrar un impacto de la influencia de la recreación; no quiere decir que los resultados fueron negativos o que no se realizó un buen diseño metodológico.

En Costa Rica, a la fecha, no se conoce algún estudio con similares alcances metodológicos que haya trabajado con población adolescente en contextos educativos la variable de bienestar subjetivo, por lo que este estudio es pionero y contribuirá a futuras investigaciones en el campo de la recreación.

Entre las limitaciones encontradas, se puede señalar que no se logró demostrar un efecto estadísticamente significativo en las variables analizadas a partir de la aplicación de un tratamiento recreativo basado en la educación no formal. Este resultado pudo estar influenciado por la buena condición encontrada en las variables analizadas, por la cantidad de sesiones (10 sesiones, una por semana de 40 minutos) que no logró desarrollar una continuidad en el proceso, porque no se realizó un diagnóstico sobre gustos e intereses de la población y porque el programa de danza urbana se ejecutó dentro del horario de clases.

A modo de recomendación se sugiere que para próximas investigaciones, las personas investigadoras tomen en cuenta que la investigación en el campo de la recreación puede realizarse desde cualquier enfoque (cuantitativo o cualitativo); sin embargo, según sea la formación disciplinaria base de la persona investigadora el diseño mixto puede enriquecer la discusión.

Es importante aproximarse a poblaciones que presenten condiciones de riesgo o prioritarias previamente identificadas, en las cuales se pueda observar una mejoría de su condición inicial, o bien, a poblaciones que realicen poca o nula actividad recreativa. Si se realizan programas de recreación en el contexto escolar se sugiere que se ejecuten dentro de la institución pero fuera del horario, y que las personas participantes sean quienes se matriculen voluntariamente en las actividades ofrecidas. 
Al trabajar con personas adolescentes es necesario contemplar un proceso diagnóstico previo, que permita tomar en cuenta los gustos e intereses recreativos de la población participante para establecer un programa de recreación adecuado, de tal manera que exista una predisposición y motivación intrínseca para participar activamente en las actividades.

Al diseñar y planificar un programa recreativo es relevante asegurarse de conocer la disponibilidad de las personas para definir la fecha, la hora, el lugar y las actividades por realizar, así como la institución u organización.

\section{Agradecimientos}

Un especial agradecimiento a la Dra. Judith Jiménez Díaz, la M.Rec. María Ester Molina Flores y la M.Rec. Karla Chaves Castro por su apoyo y acompañamiento durante la realización de la investigación. Al CTP Ing. Mario Quirós Sasso por su apertura, en especial al Prof. Gerardo Sibaja y la Prof. Ivannia Brenes, al estudiantado por su valiosa participación y a Brayan Fonseca por su excelente desempeño como breakdancer.

\section{Referencias}

Atienza, Francisco., Pons, Diana., Balaguer, Isabel. y García-Merita, Marisa. (2000). Propiedades psicométricas de la Escala de Satisfacción con la vida en adolescentes. Psicothema, 12(2), 314-319. Recuperado de http://www.psicothema.com/psicothema.asp?id=296

Barrantes-Brais, Kristy. y Ureña-Bonilla, Pedro. (2015). Bienestar psicológico y bienestar subjetivo en estudiantes universitarios costarricenses. Revista Intercontinental de Psicología y Educación, 17(1), 101-123. Recuperado de https://www.redalyc.org/pdf/802/80242935006.pdf

Bello, Anyerson., Puerta, Erika. y Teherán, Neryle. (2016). La danza urbana como perspectiva de vida: Una experiencia con el grupo Ad Crew [Informe del video documental Caminos, la danza urbana como perspectiva de vida]. Universidad de Cartagena, Colombia. Recuperado https://repositorio.unicartagena.edu.co/bitstream/handle/11227/5289/LA\%20DANZA\%2 0URBANA\%20COMO\%20PERSPECTIVA\%20DE\%20VIDA.pdf?sequence=1

Brinnitzer, Evelina. (2003). Adolescencia, pobreza y tiempo libre en mujeres y varones. Revista de Estudios de la Mujer, 8(1), 221-244. Recuperado de https://bit.ly/3krQRON

Cabalé, Elizabeth. y Rodríguez, Gabriel. (2017). Educación no Formal: potencialidades y valor social. Revista Cubana de Educación Superior, 36(1), 69-83. Recuperado de http://scielo.sld.cu/pdf/rces/v36n1/rces07117.pdf 
Carballeira, Mónica., González, José-Ángel. y Marrero, Rosario. (2015). Diferencias transculturales en bienestar subjetivo: México y España. Anales de Psicología, 31(1), 199-206. doi: http://dx.doi.org/10.6018/analesps.31.1.166931

Connolly, Mary., Quin, Edel. y Redding, Emma. (2011). Dance 4 your life: Exploring the health and wellbeing implications of a contemporary dance intervention for female adolescents. Research in Dance Education, 12(1), 53-66. doi: https://doi.org/10.1080/14647893.2011.561306

Consejo Nacional de Niñez y Adolescencia. (2009). Política nacional para la niñez y la adolescencia. Costa Rica 2009-2021. Recuperado de https://www.corteidh.or.cr/sitios/Observaciones/2/AnexoVI.pdf

Daykin, Norma., Orme, Judy., Evans, David., Salmon, Debra., McEachran, Malcolm. y Brain, Sarah. (2008). The impact of participation in performing arts on adolescents health and behavior: A systematic review of the literature. Journal of Health Psychology, 13(2), 251 264. doi: https://doi.org/10.1177/1359105307086699

DeGraaf, Donald., Jordan, Debra. y DeGraaf, Kathy. (2010). Programming for parks, recreation, and leisure services: a servant leadership approach ( $3^{\text {rd }}$ ed.). State College, Pennsylvania: Venture Publishing, Inc. Recuperado de https://www.sagamorepub.com/sites/default/files/2018-07/programmingebooklookinsideopt.pdf

Godoy-Izquierdo, Débora., Martínez, Antonio. y Godoy, Juan. (2008). La escala de balance afectivo. Propiedades psicométricas de un instrumento para la medida del afecto positivo y negativo en población española. Clínica y Salud, 19(2), 157-189. Recuperado de https://bit.ly/3dXkpS5

Gomes, Christianne. (2014). El ocio y la recreación en las sociedades latinoamericanas actuales. Polis, Revista Latinoamericana, 13(37), 363-384. Recuperado de https://www.redalyc.org/pdf/305/30531107020.pdf

Gutiérrez, Melchor., Tomás, José. y Calatayud, Pablo. (2017). Influencia del clima motivacional en educación física sobre las metas de logro y la satisfacción con la vida de los adolescentes. Retos: Nuevas tendencias en Educación Física, Deportes y Recreación, (31), 157-163. doi: https://doi.org/10.47197/retos.v0i31.49421

Hincapié, Astrid. (2014). El Hip Hop: Una práctica corporal que territorializa la ciudad de Medellín. Poiésis, 8(14), 385-402. Recuperado de http://www.portaldeperiodicos.unisul.br/index.php/Poiesis/article/download/2619/1907

Instituto Colombiano del Deporte (Coldeportes). (2006). Beneficios de la recreación: Por una apropiación comunitaria, recreativa y participativa, de los juegos deportivos. Bogotá, Colombia: Instituto Colombiano del Deporte. Recuperado de https://blog.utp.edu.co/areaderecreacionpcdyr/files/2012/07/Beneficios-de-laRecreaci\%c3\%b3n.pdf 
Laca, Francisco. (2015). El largo camino hacia el estudio científico de la satisfacción con la vida y el bienestar subjetivo. Pensamiento Americano, 8(15), 27-40. Recuperado de http://oaji.net/articles/2016/2339-1473527005.pdf

Lara, Laura. (2017). Adolescentes latinoamericanos en España: Aculturación, autonomía conductual, conflictos familiares y bienestar subjetivo. Universitas Psychologica, 16(2), 1-11. oi: https://doi.org/10.11144/Javeriana.upsy16-2.alea

Manotas, Reinaldo. y Ovalles, Liana. (2017). Impacto social y cultural del Hip Hop. Revista Convicciones, 4(7), 159-164. Recuperado de https://www.fesc.edu.co/Revistas/OJS/index.php/convicciones/article/view/129

Merino, Laura. (2015). El Hip-Hop como herramienta socioeducativa durante la adolescencia (Tesis de Licenciatura). Universidad de Valladolid. Recuperado de http://uvadoc.uva.es/handle/10324/14414

Organización Mundial de la Salud (OMS). (13 de diciembre, 2018). Adolescentes: Riesgos para la salud y soluciones. Recuperado de https://www.who.int/es/news-room/factsheets/detail/adolescents-health-risks-and-solutions

Padilla, Carmen. y Coterón, Javier. (2013). ¿Podemos mejorar nuestra salud mental a través de la danza?: una revisión sistemática. Retos. Nuevas tendencias en Educación Física, Deportes y Recreación, (24), 167-170. doi: https://doi.org/10.47197/retos.v0i24.34556

Philipsson, Anna., Duberg, Anna., Möller, Margareta. y Hagberg, Lars. (2013). Cost-utility analysis of a dance intervention for adolescent girls with internalizing problems. Cost Effectiveness and Resource Allocation, 11(4), 1-9. doi: https://doi.org/10.1186/1478$\underline{7547-11-4}$

Quin, Edel., Frazer, Lucy., y Redding, Emma. (2007). The health benefits of creative dance: Improving children's physical and psychological wellbeing. Education \& Health, 25(2), 3133. Recuperado de https://bit.ly/3mjskfg

Ramos-Díaz, Estibaliz., Rodríguez-Fernández, Arantzazu. y Antonio-Aguirre, Iratxe. (2017). El autoconcepto y el bienestar subjetivo en función del sexo y del nivel educativo en la

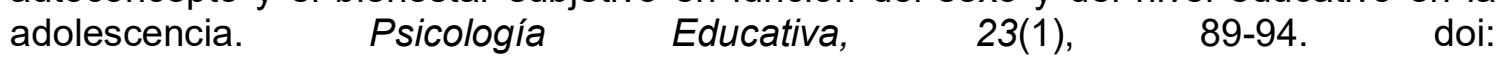
https://doi.org/10.1016/j.pse.2017.05.005

Rodríguez, Alberto. e Iglesias, Lucía. (2014). La cultura Hip Hop: Revisión de sus posibilidades como herramienta educativa. Teoría de la Educación. Revista Interuniversitaria, 26(2), 163-182. Recuperado de https://revistas.usal.es/index.php/11303743/article/view/teoredu2014261163182

Rodríguez-Fernández, Arantzazu., Ramos-Díaz, Estibaliz., Fernández-Zabala, Arantza., Goñi, Eider., Esnaola, Igor. y Goñi, Alfredo. (2016). Contextual and psychological variables in a descriptive model of subjective well-being and school engagement. International Journal of Clinical and Health Psychology, 16(2), 166-174. doi: https://doi.org/10.1016/j.ijchp.2016.01.003 
Romero, Andrea. (2010). A pilot test of the Latin active Hip Hop intervention to increase physical activity among low-income Mexican-American adolescents. American Journal of Health Promotion, 26(4), 208-211. doi: https://doi.org/10.4278/ajhp.090123-arb-24

Romero, Cecilia. (2015). La recreación en el fomento de la resiliencia. Revista de Ciencias de la Actividad Física, 16(1), 63-80. Recuperado de https://www.redalyc.org/pdf/5256/525652730008.pdf

Salazar, Carmen. (2017). Recreación (2a ed.). San José: Editorial de la Universidad de Costa Rica.

Sánchez, Marilyn. (2020). Recreación artística y bienestar subjetivo en estudiantes adolescentes del Colegio Técnico Profesional Ing. Mario Quirós Sasso (Tesis de Maestría). Universidad de Costa Rica, Costa Rica.

Sandoval, José. (2014). La subjetividad en el enfoque del desarrollo: Calidad de vida, bienestar subjetivo y capacidades. Límite: Revista Interdisciplinaria de Filosofía y Psicología, 9(30), 35-48. Recuperado de https://www.revistalimite.cl/index.php/limite/article/view/19

San Martín, Juan. (2011). Bienestar subjetivo en adolescentes: Relación entre satisfacción vital, autoestima, esperanza y apoyo social (Tesis de Maestría). Universidad de Concepción, Chile. Recuperado de http://repositorio.udec.cl/handle/11594/1779

Schwender, Tina., Spengler, Sarah., Oedl, Christina. y Mess, Filip. (2018). Effects of dance interventions on aspects of the participants' self: A systematic review. Frontiers in Psychology, 9. doi: https://www.frontiersin.org/articles/10.3389/fpsyg.2018.01130/full

Zapata, Adriana. (2016). Bienestar psicológico y bienestar social en estudiantes universitarios que realizan danzas folklóricas (Tesis de Licenciatura) Pontificia Universidad Católica del Perú. Recuperado de http://tesis.pucp.edu.pe/repositorio/handle/20.500.12404/7712 


\section{Anexos}

\section{A. Escala de Satisfacción con la Vida}

A continuación encontrará varias afirmaciones con las cuales usted puede estar de acuerdo o en desacuerdo con ellas. Por favor, utilice la siguiente escala de 1 a 7 para indicar el grado en que cada afirmación se aplica en su caso.

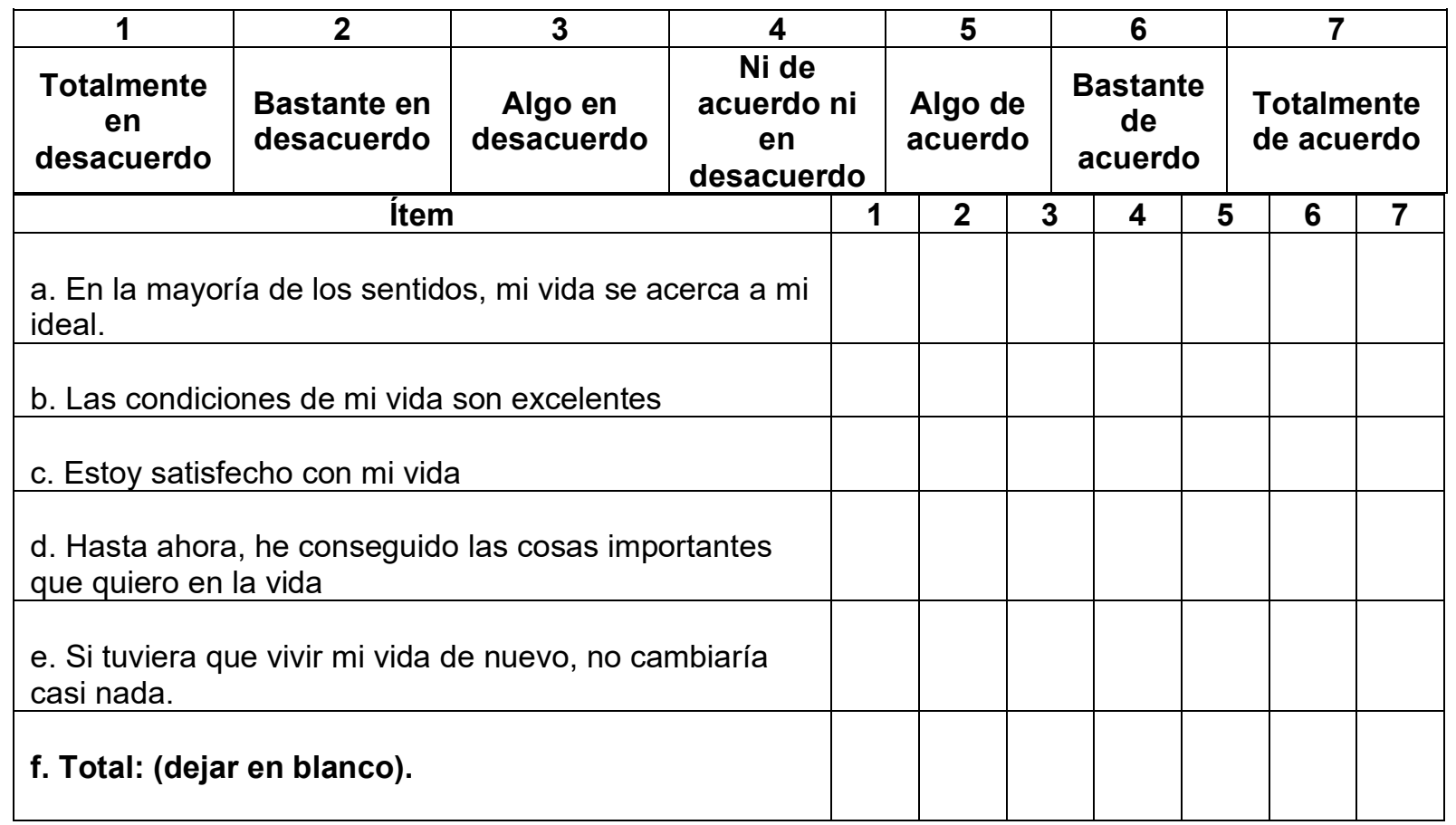




\section{B. Escala de Balance Afectivo}

A continuación se le presentan una serie de enunciados, marque con una $x$ la opción que más representa su opinión y que ha sentido en la última semana:

\begin{tabular}{|l|l|l|l|}
\hline \multicolumn{1}{|c|}{ İ́m } & \multicolumn{1}{|c|}{$\begin{array}{c}\text { Poco o } \\
\text { nunca (1) }\end{array}$} & A veces (2) & $\begin{array}{c}\text { Mucho o } \\
\text { generalmente } \\
\text { (3) }\end{array}$ \\
\hline 1. ¿Se ha sentido molestado(a) por alguien? & & & \\
\hline 2. ¿Se ha sentido muy solo(a) o distante de la gente? & & & \\
\hline 3. ¿Ha sentido que las cosas iban como usted quería? & & & \\
\hline 4. ¿Se ha sentido muy preocupado(a)? & & & \\
\hline $\begin{array}{l}\text { 5. ¿Se ha sentido contento(a) por tener gente con la } \\
\text { que contar o hacer algo? }\end{array}$ & & & \\
\hline $\begin{array}{l}\text { 6. ¿Ha tenido miedo de lo que pudiera suceder? } \\
\text { 7. ¿Se ha sentido particularmente estimulado(a) o } \\
\text { interesado(a) por algo? }\end{array}$ & & \\
\hline 8. ¿Se ha sentido deprimido(a) o muy infeliz? & & \\
\hline 9. ¿Se ha sentido lleno(a) de energía? & & \\
\hline 10. ¿Se ha sentido muy cansado(a)? & & \\
\hline $\begin{array}{l}\text { 11. ¿Se ha sentido usted muy nervioso(a), agobiado(a) } \\
\text { o tenso(a)? }\end{array}$ & & & \\
\hline 12. ¿Ha sentido que se estaba divirtiendo mucho? & & & \\
\hline 13. ¿Se ha sentido muy alegre o feliz? & & \\
\hline 14. ¿Se ha sentido con ganas de llorar? & & \\
\hline $\begin{array}{l}\text { 15. ¿Se ha sentido eufórico(a) (muy contento(a), } \\
\text { dichoso(a))? }\end{array}$ & & & \\
\hline 16. ¿Se ha sentido seguro(a) respecto al futuro? & & & \\
\hline 17. ¿Se ha sentido aburrido(a)? & & & \\
\hline $\begin{array}{l}\text { 18. ¿Se ha sentido contento(a) o satisfecho(a) por } \\
\text { haber logrado algo? }\end{array}$ & & & \\
\hline
\end{tabular}


Revista indizada en

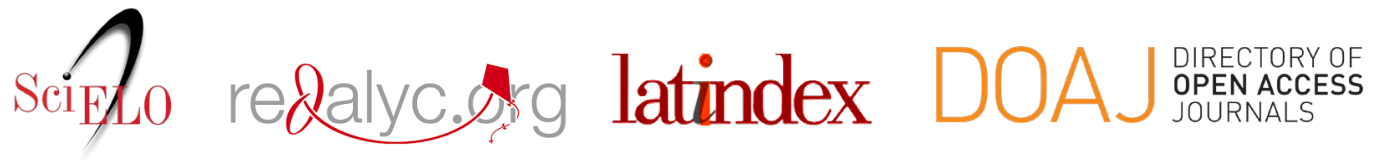

Distribuida en las bases de datos:

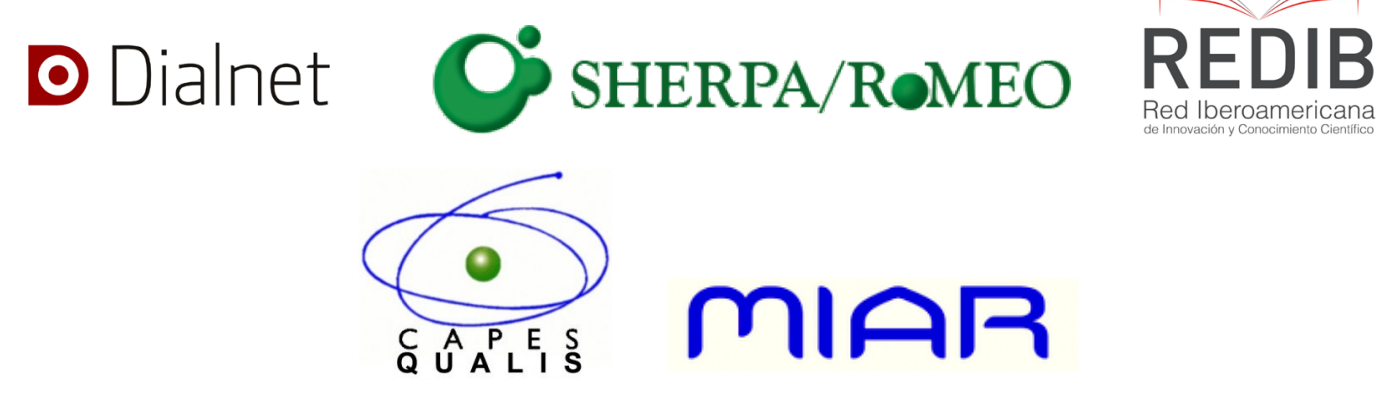

